\title{
Parametric Models of Facade Designs of High-Rise Residential Buildings
}

\author{
Yuchen Sharon Sung and Yingjui Tseng
}

\begin{abstract}
High-rise residential buildings have become the most mainstream housing pattern in the world's metropolises under the current trend of urbanization. The facades of high-rise buildings are essential elements of the urban landscape. The skins of these facades are important media between the interior and exterior of high- rise buildings. They not only connect between users and environments, but also play an important functional and aesthetic role. This research involves a study of skins of high-rise residential buildings using the methodology of shape grammar to find out the rules which determine the combinations of the facade patterns and to analyze the patterns' parameters using software Grasshopper. We chose a number of facades of high-rise residential buildings as source to discover the underlying rules and concepts of the generation of facade skins.
\end{abstract}

This research also provides the rules that influence the composition of facade skins. The items of the facade skins, such as windows, balconies, walls, sun visors and metal grills are treated as elements in the system of facade skins. The compositions of these elements will be categorized and described by logical rules; and the types of high-rise building facade skins will be modelled by Grasshopper. Then a variety of analyzed patterns can also be applied on other facade skins through this parametric mechanism. Using these patterns established in the models, researchers can analyze each single item to do more detailed tests and architects can apply each of these items to construct the facades for their other buildings through various combinations and permutations. The goal of these models is to develop a mechanism to generate prototypes in order to facilitate design process of various facade skins.

Index Terms-Facade skin, grasshopper, high-rise residential building, shape grammar.

\section{INTRODUCTION}

Since 1952, when the first curtain wall was installed in New York City in the Lever House building by Skidmore, Owings and Merrill, the concept of facade skin was gradually applied to high-rise buildings. Nowadays it is considered as an aspect of buildings which is studied and designed independently.

Due to the trend of global warming the issue of sustainability impacted the composition of facade skins since the last decade. It conveyed more complicated functions and installations on the envelopes of buildings, such as heat

\footnotetext{
Manuscript received November 26, 2014; revised February 27, 2015 This work was supported in part by Wang Architects and Associates, Taichung, Taiwan. We also had many interesting discussions with the designers and architects of Wang Architects and Associates and received many useful feedbacks during the process of this study.

The authors are with Architecture Department at Feng Chia University, Taichung, $40724 \quad$ Taiwan (e-mail: ycsung@fcu.edu.tw, v6yourvoice@yahoo.com.tw).
}

insulation, ventilation system or renewable energy panels. Along with the innovation of ever-changing technologies the facade skins have been developing into an intricate but interesting multilayered system [1]. The elements of facade skins, such as doors, windows and balconies can be operated as parametric factors to derive the optimal solutions of facade patterns. The most valuable part is a parametric system that provides a mutual and vital framework for researchers and designers to go deeper into any issues, such as ecology, aesthetics and sustainability. The literature related to the issue of sustainability and ecology for building facades has been published both in many conference-proceedings and journals in the last decade. Therefore this research will focus on how to provide a parametric system of facade skins for high-rise residential buildings which can be analyzed by rules and by software Grasshopper.

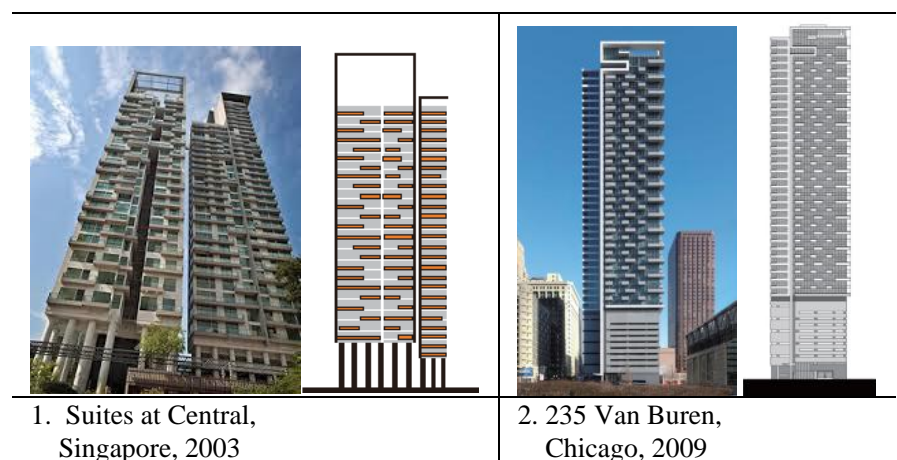

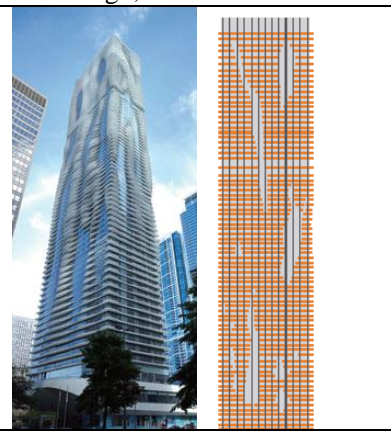

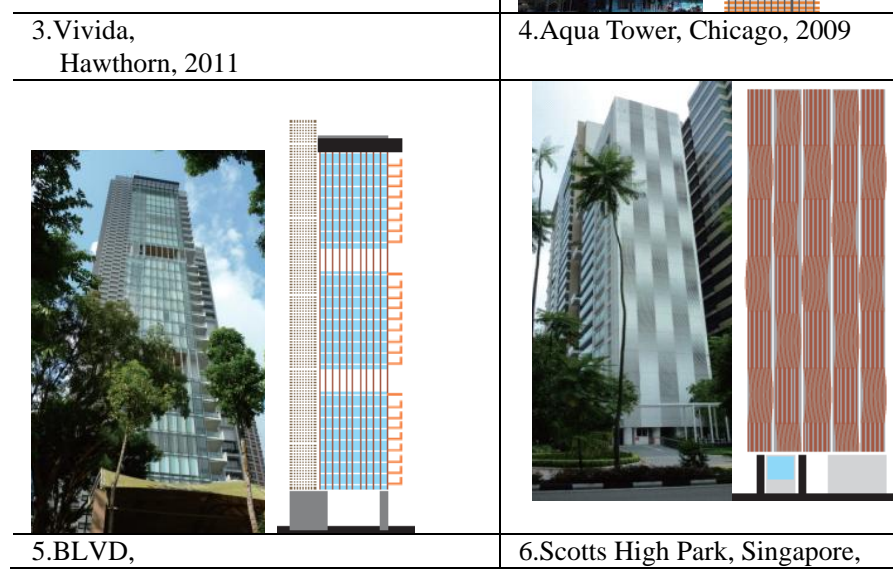




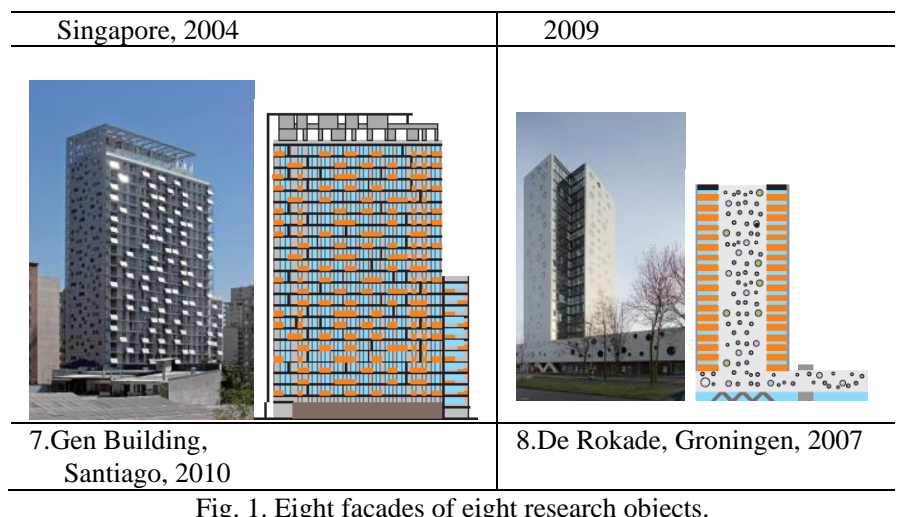

Fig. 1. Eight facades of eight research objects.

\section{RESEARCH OBJeCtS}

In this research facades from eight high-rise residential buildings which were completed around the last decade and complied with the conditions of being higher than 10 floors and being located at the central area of metropolises are chosen. The pictures and information of these research objects are listed in Fig. 1.

The main reason to raise up these eight research objects is because they stand out for the vital items of their facade skins. These vital items on the facade patterns can be sorted as balconies, skin patterns, windows, sunshade elements and renewable solar energy panels etc. Of these research objects two are located in America, three in Asia and three in Europe. We do not make it mandatory that all research objects should be in the same area or all research facades should be facing the same direction, because the elements of these facades, no matter where they are from or how they are formed, can be shaped and adjusted to meet the needs of the building at the site. Architects, designers and researchers are able to decide facade patterns and items for their facade design or researches. There should be no limits for case studies in design studios or for research objects in this field. In a general global sense these vital facade items could reveal the architects' aesthetics, the climate's conditions of the environment and the orientation of any building facade in any metropolis.

\section{SHAPE GRAMMAR}

The methodology, shape grammar, is encouraged by Stiny and Gips (1972), who introduce a powerful methodology to explain a great variety of architectural forms by rules. Besides being able to show highly structured geometric form, irregular fractals such as buildings and urban designs can also be efficiently described by shape grammar. There has been a lot of literature on building facades regarding shape grammar. The review here primarily focuses on recent works involving combinations of skin patterns and extractions of features of facade elements. A facade segmentation framework is modeled in a hierarchy by 2D images of a rectangular area of a contour of facades. The operation recursively split the image vertically and horizontally into two parts by maximizing the entire length of regular rims until the content of the facade hypothesis matched to a given model [2]. It can also derive $3 \mathrm{D}$ models when we develop the procedural modelling rules of shape grammar with 3D image analyses to a hierarchical facade subdivision. It helps us to build a rule base for procedural facade modelling technology [3], [4]. A kind of stochastic semantic grammars is applied to recompose facades from image data by horizontal and vertical cuts [5]. The process can be operated based on reversible jump Markoy Chain Monte Carlo (rjMCMC) to adapt the application of derivation paces in the construction of the tree diagram [6], [7]. The elements of facades can be extracted by a formal grammar and described in the form of a derivative tree analyses. This modelling technology is also based on images of facades. The images of facades can be subdivided and encoded as shape systems of tree analyses through systematic models which can also be reversed [8].

The facades of high-rise buildings are often largely governed by concatenated interlaced grids. Therefore an adaptive facade partition can be performed reversely to generate a hierarchical representation of the facade skin in a grid system as well as in a framework. It shows that the concept of adaptive partitioning is also applicable to flexible analyses of image facades [9]. Even an irregular lattice on facades can be inferred by combining low-level classifiers with mid-level parses by grammars. The irregular lattice keeps the logical framework of the facade while providing the possibilities of varieties of elements of facade patterns [10]. These concepts inspire us to set up a classified form framework of building facades by shape grammar. And the elements on the facade patterns can be extracted by rules, as the features of these elements are seen as the genes that form the facade patterns. Then the rules of forming these elements will be tried to develop into the parametric factors of forming facade skins.

\section{Combinations Of Basic Forms}

The basic form of a high-rise building can be divided in three ways: 1. top, middle and base; 2. top and middle; 3.middle and base; 4 . whole. Besides these there will be many possibilities of free forms as the Fig. 2 below.

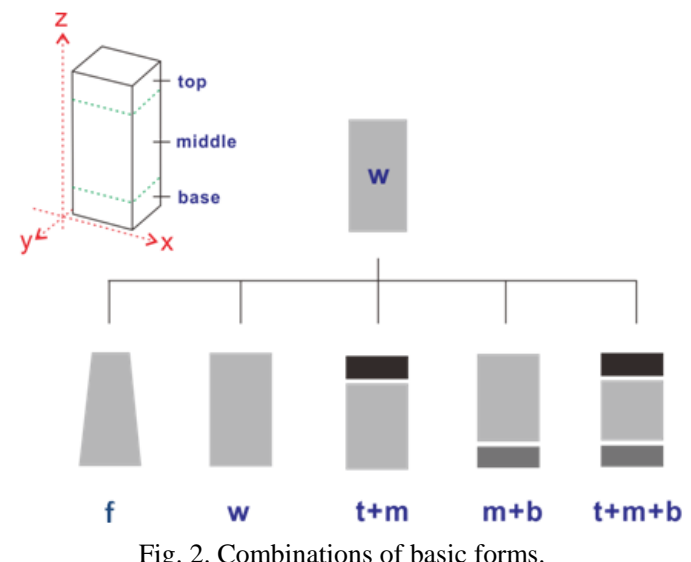

If a facade is set on $\mathrm{X}$ - and Z- coordinate system, a system of rules can be built for the sketch of a two- dimension facade as showed in Fig. 2 below. According to this system of rules, the combinations of facade skins for high-rise residential buildings can be described through 10 rules that derive the basic forms of these 8 research objects or many other high-rise buildings in Fig. 3. 
RO Initial shape

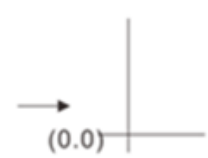

R1 A whole volume
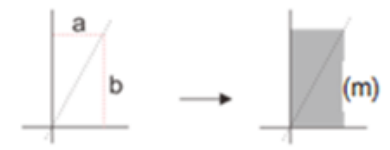

R2 To divide top from a whole

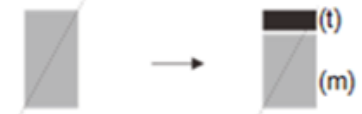

R3 To divide base from a whole
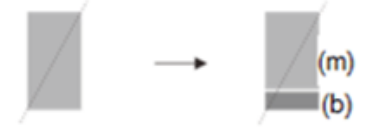

R4 Vertical division , $M$ section, $M \geq 2$
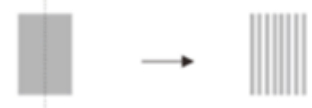

R5 Horizontal division, $\mathrm{N}$ section, $\mathrm{N} \geq 2$

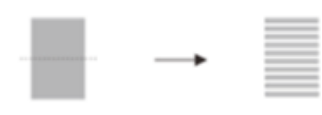

R6 To adjust proportion of sections

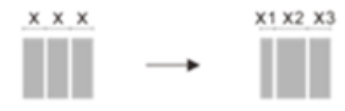$$
\begin{aligned}
& \mathbf{y} \\
& \mathbf{y} \\
& \mathbf{y}
\end{aligned}
$$
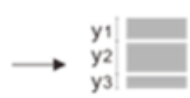

R7 To generate stripes

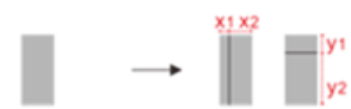

R8 To generate columns

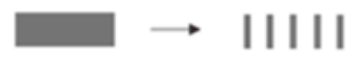

R9 To delete section units

R10 To generate extra section units

Fig. 3. Rules for deriving the sketches of the combinations of these high-rise residential buildings.

Rule 0 is the initial shape and is used to set an X- and Zcoordinate system. Rule 1 is to generate a whole volume of a basic form. Rule 2 and 3 are set for separating the parts of a basic form, such as top, middle and base and their combinations. Rule 4 and 5 are used to divide vertical and horizontal sections on a basic form. Rule 6 is to adjust the measurement of the sections, if the basic combinations are divided. Rule 7 is to generate vertical or horizontal stripes on the facade skins. Rule 8 is to generate columns on the facades Rule 9 is to delete section units from the facades. Rule 10 is to generate an extra section unit, if it should be added. Then the rules are compiled and the diagrams of the composition of the 2D forms for these eight high-rise residential buildings are developed. Thus, the 2D forms of these eight residential buildings are disassembled and analyzed through these ten rules by a tree diagram in Fig. 4. After the sketches of the facades of these research objects have been analyzed by rules, the patterns of elements and items, such as openings and balconies on the facades can be extracted and modelled.

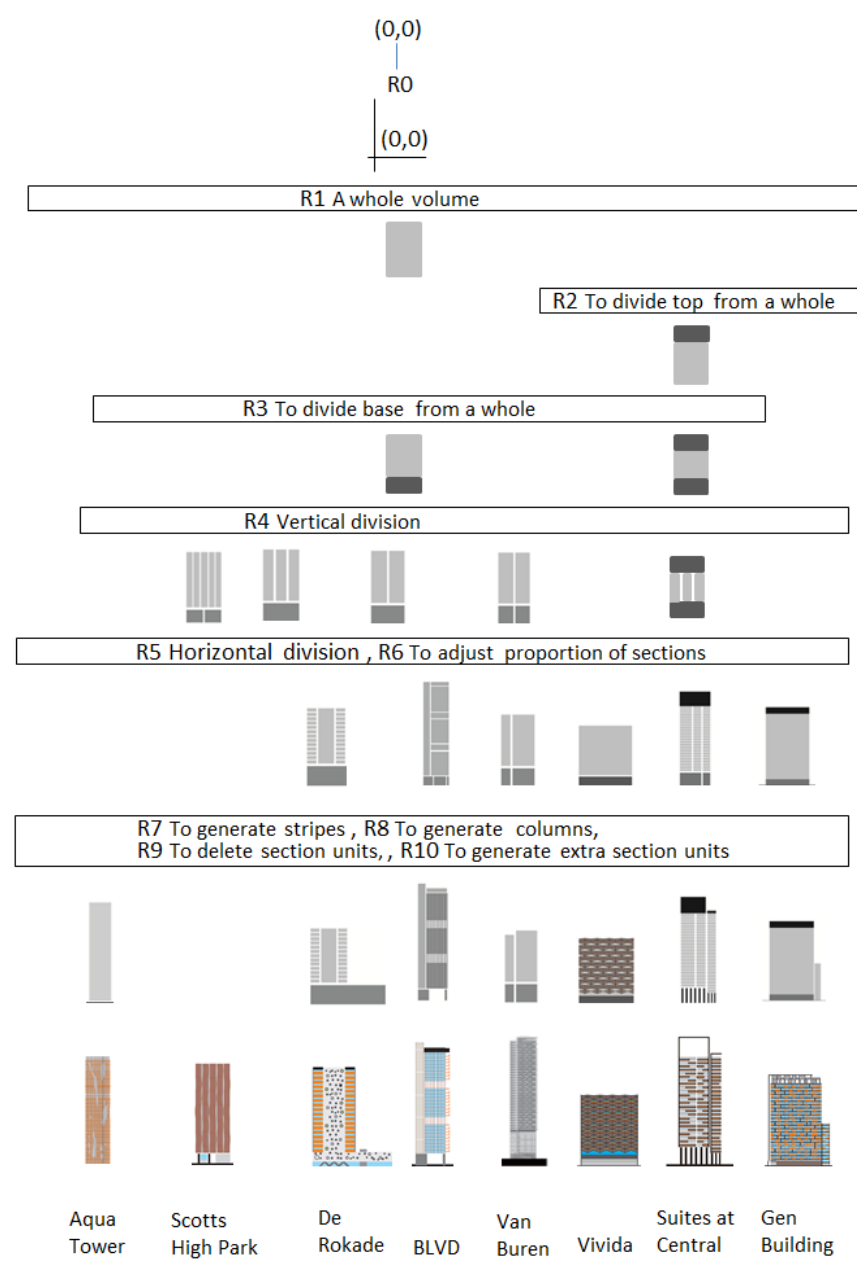

Fig. 4. The tree diagram of the combinations of the basic forms of these eight research objects.

\section{EXTRACTION THE PATTERNS OF OPENINGS From RESEARCH OBJECTS}

The purpose of extracting the patterns of facade elements and items is to find the features of the facades and the logical parametric factors of these elements which can be modelled by software Grasshopper. These features are comparable to the genes on biology and are the source to produce the similar or superior offspring and to generate various proposals on facade in design. Thus, a large amount of genetic compositions can be processed in a very efficient time span and may contain some unexpected and unpredictable forms [11]. As soon as these parametric factors are extracted and modelled, they can also be applied to the design of the facade skins of other buildings. Furthermore they can be studied as a key for shaping a single facade item combined with other knowledge and technology. For example, an extracted louver system can be checked and modified because of climate conditions of its surrounding, ecological technologies and 
even aesthetic reasons. And so forth a green wall, a solar panel and a ventilation system can also be adjusted by a similar approach.

According to this notion of extraction of the patterns of openings, research objects 2,7 and 8 are chosen here. The parametric factors for balconies, solar panels and window systems of these high-rise residential buildings are extracted in Fig. 5. The others are shown and combined with the analyses by Grasshopper in Table I.

Although the concept of grammar has been used to model architectural patterns both of floor plans and facades in the related field of shape grammar, the extraction of their parametric factors and modelling by Grasshopper is still quite new. In this research we try to extract the patterns of openings from the facades of our eight research objects and to perceive what parametric factors can be operated in Grasshopper. These patterns of openings are classified into four kinds below: A. balcony and terrace, B. solar, louver and perforated panel, C. window, D. others.

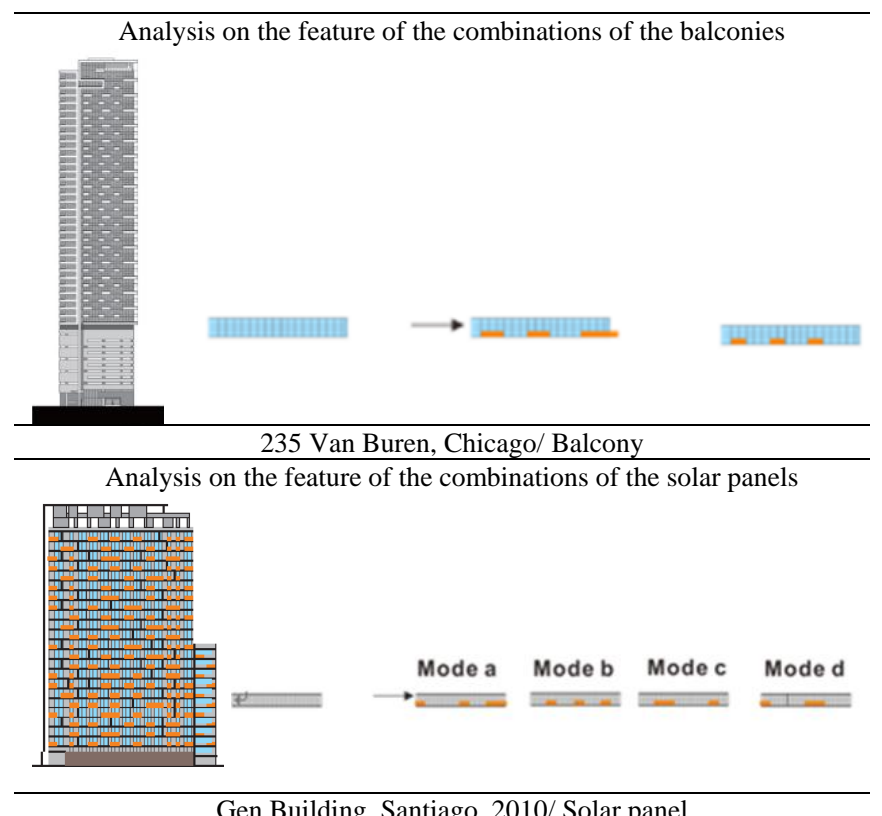

Analysis on the feature of the combinations of the round windows

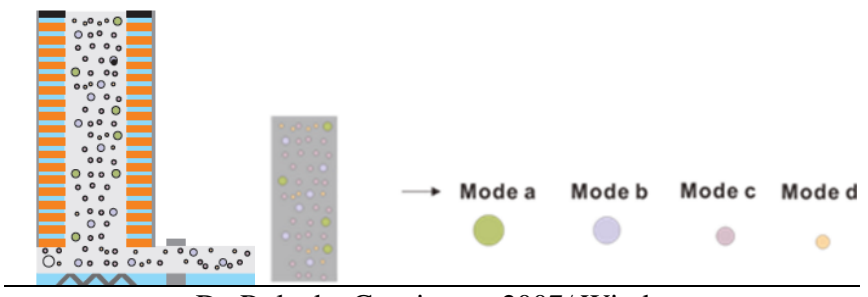

De Rokade, Groningen, 2007/ Window

Fig. 5. Feature analyses: Extractions of the parametric factors for balcony, solar panel and window systems from the research objects.

\section{PARAmetric ANALyses of These FaCAde Skins by GRASSHOPPER}

After a parametric factor of a facade item is extracted, then it can be operated in the digital model by software Grasshopper according to the grammar rules of the combinations, forms and types of this facade item. For example, A1 Balcony item, there are 5 types of combinations of balconies on the facade skin of our research object 1 : Suites at Central.

\section{A. Analysis and Flowchart}

The feature of its main facade is analyzed and then a flow chart is developed according to the logic and grammar rules for this feature.

\section{B. Geometric and Operational Rules of Grasshopper}

Then its geometric and operational rules can be built up by Grasshopper. The common factors of a building such as the numbers of stories of a building and the height of one story should be set first. Afterwards the parametric factors such as the numbers of balconies, the width, the length and the depth of a balcony and the positon of a balcony can be set up as parameters and the measurements of these factors can be adjusted. These analyses, flowcharts and geometric rules for the eight research objects are shown in Table I.

Similarly, in Table I the A2 Balcony item there are two main combinations of balconies on the facade of 235 Van Buren building. Therefore the combinations of balconies of the odd and even stories can be adjusted through the parameters of the width, the length or the depth of a balcony. The balcony at A3 Aqua Tower depends on the climate condition which defines the curve of its balconies, so this balcony item should be able to be adjusted according to the climate condition of any site.

There are three sunshade elements and one solar panel element in the category B. The sunshade elements at B1 are staggered horizontally, so they are described at the odd and even stories correspondingly. For the feature of this sunshade element, the folding angle of these folding sunshade panels is set to be adjustable by a parameter. The vertical sunshade panel at B2 is twisted; therefore the parameter for twisting the sunshade panels should be controlled after the measurements of a vertical sunshade panel has been decided. The grid sunshade system at B3 can be adjusted in width, length or depth of the grid system. The solar elements at B4 which have two sizes of panels are installed randomly. So the first step is to set the parameters of the two sizes of panels, then to array these panels randomly. There are four different sizes of round windows at the last category in Fig. 6, therefore the parameters of the four sizes of these round windows should be set first, then they are arrayed randomly on the facade of the building.

TABLE I: PARAMETRIC ANALYSES AND MODELS OF THE FACADE ITEMS OF THE RESEARCH OBJECTS BY GRASSHOPPER A1. Balcony item: 1. Suites at Central, Singapore Extraction of the pattern

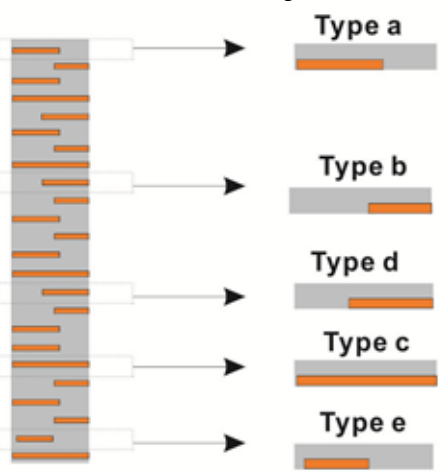

Flow Chart

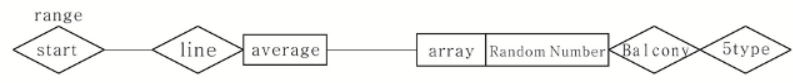




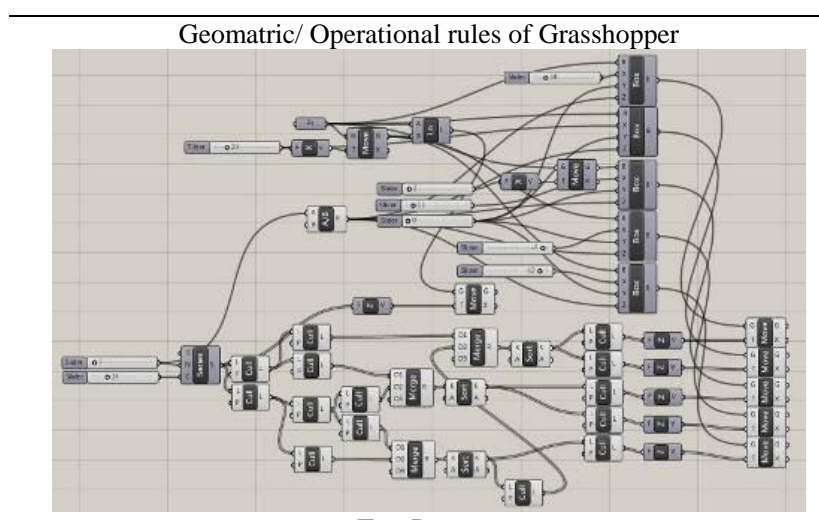

Test Patterns

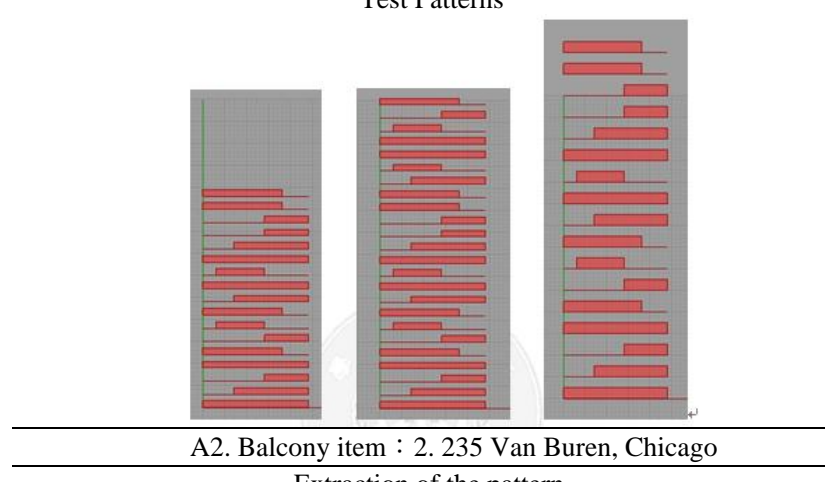
Extraction of the pattern

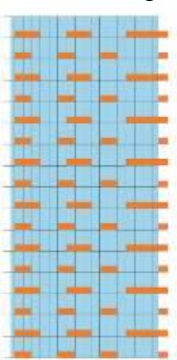

Flow Chart

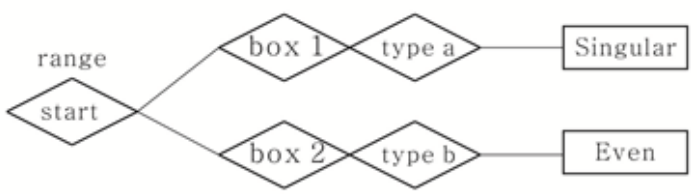

Geomatric/ Operational rules of Grasshopper

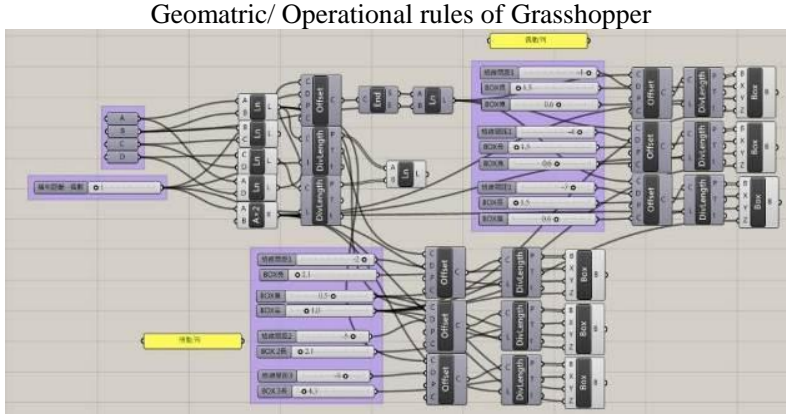

Test Patterns

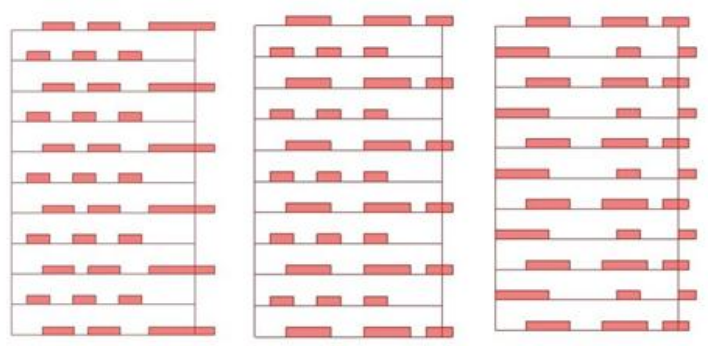

A3. Balcony item: 3. Aqua Tower, Chicago Extraction of the pattern

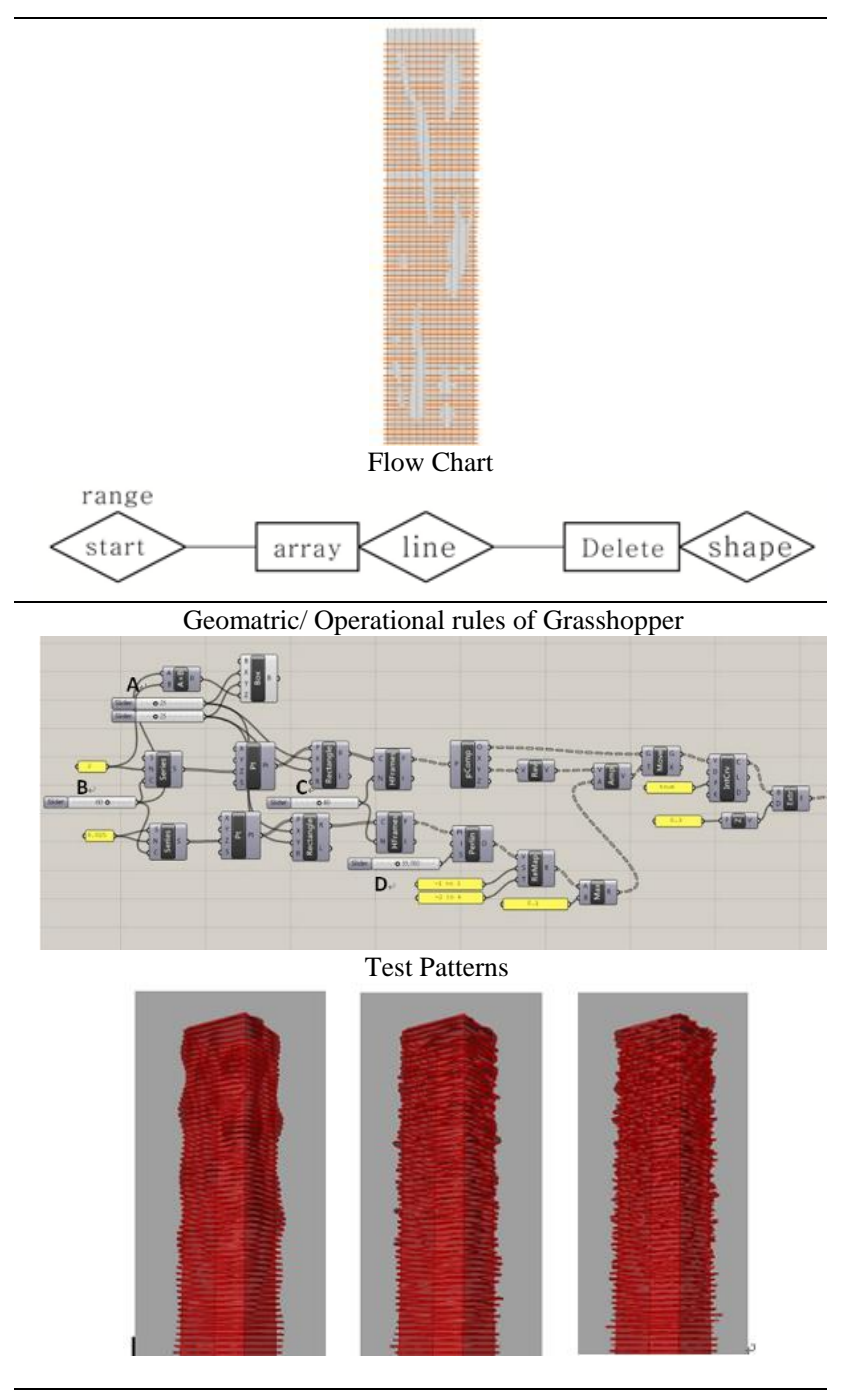

B1. Sunshade element: 4. Vivida, Hawthorn

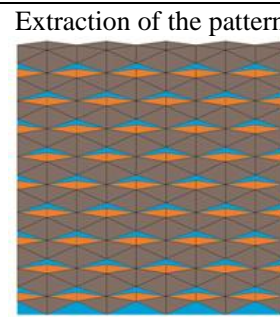

Flow Chart
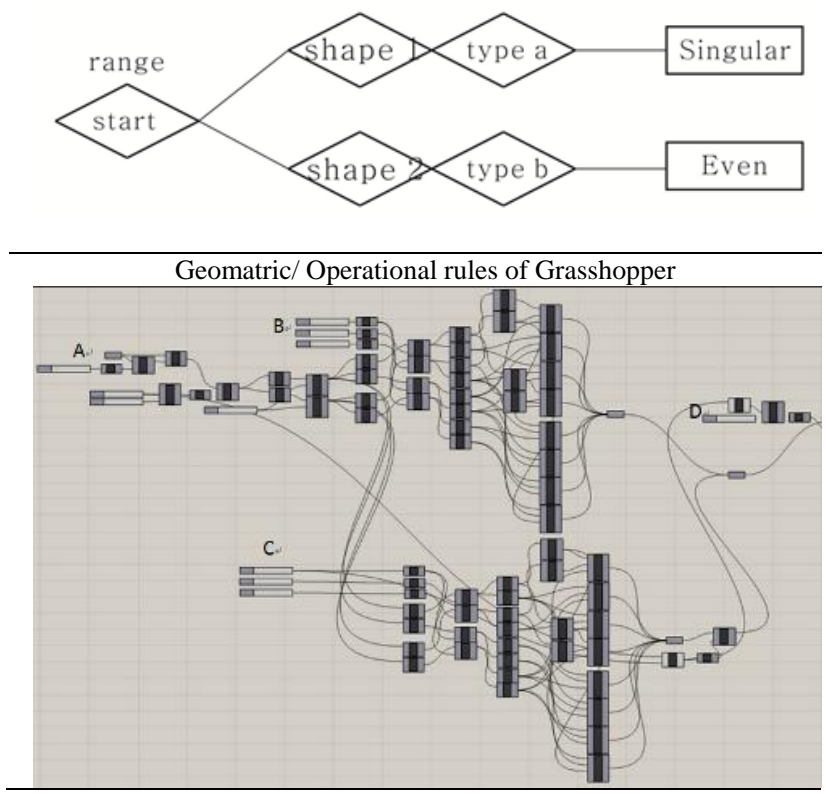


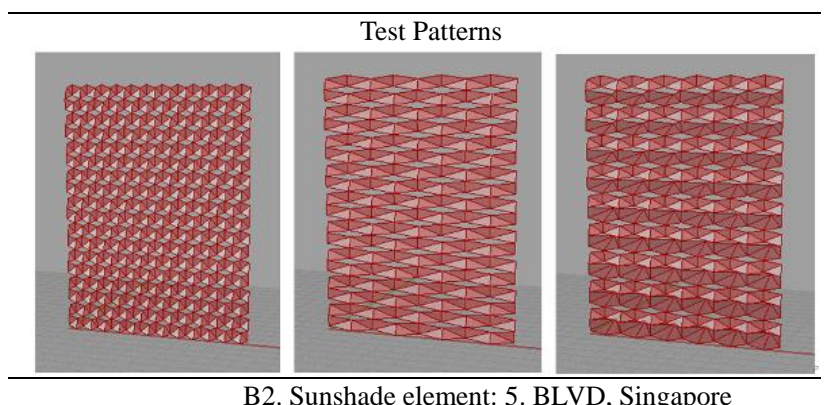

B2. Sunshade element: 5. BLVD, Singapore

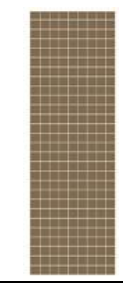
Extraction of the pattern / Flow Chart

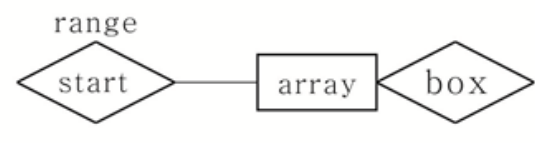

Geomatric/ Operational rules of Grasshopper

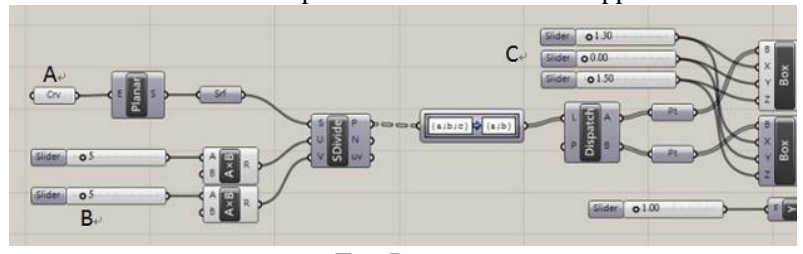

Test Patterns

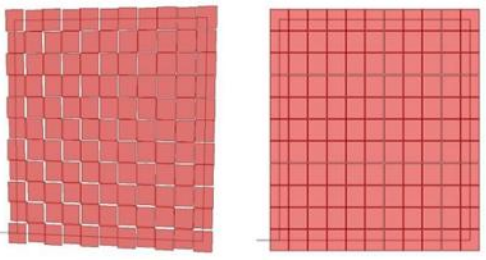

B3. Sunshade element: 6. Scotts High Park, Singapore Extraction of the pattern

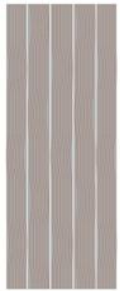

Flow Char

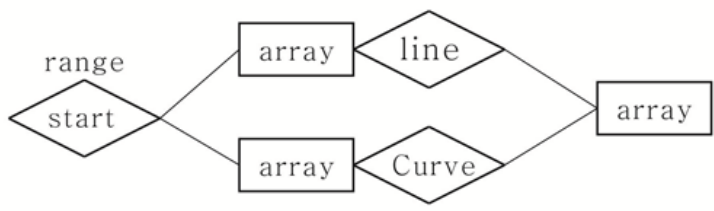

Geomatric/ Operational rules of Grasshopper
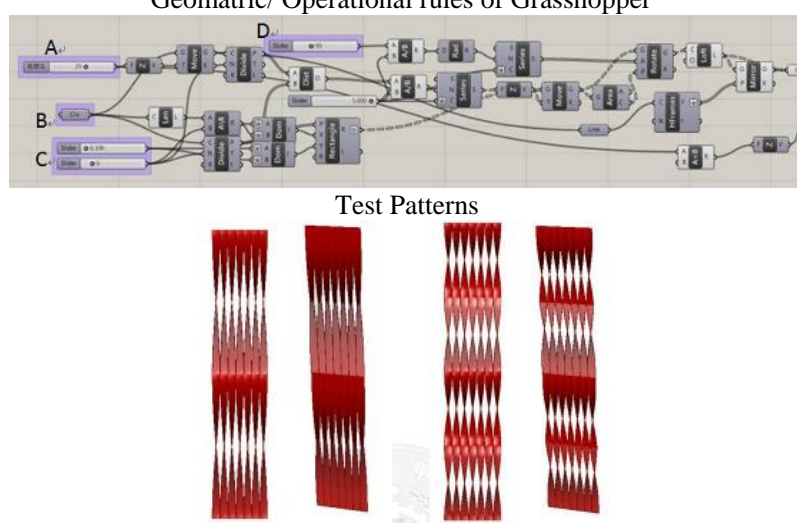

B4. Solar panel element: 7. Gen Building, Santiago Extraction of the pattern

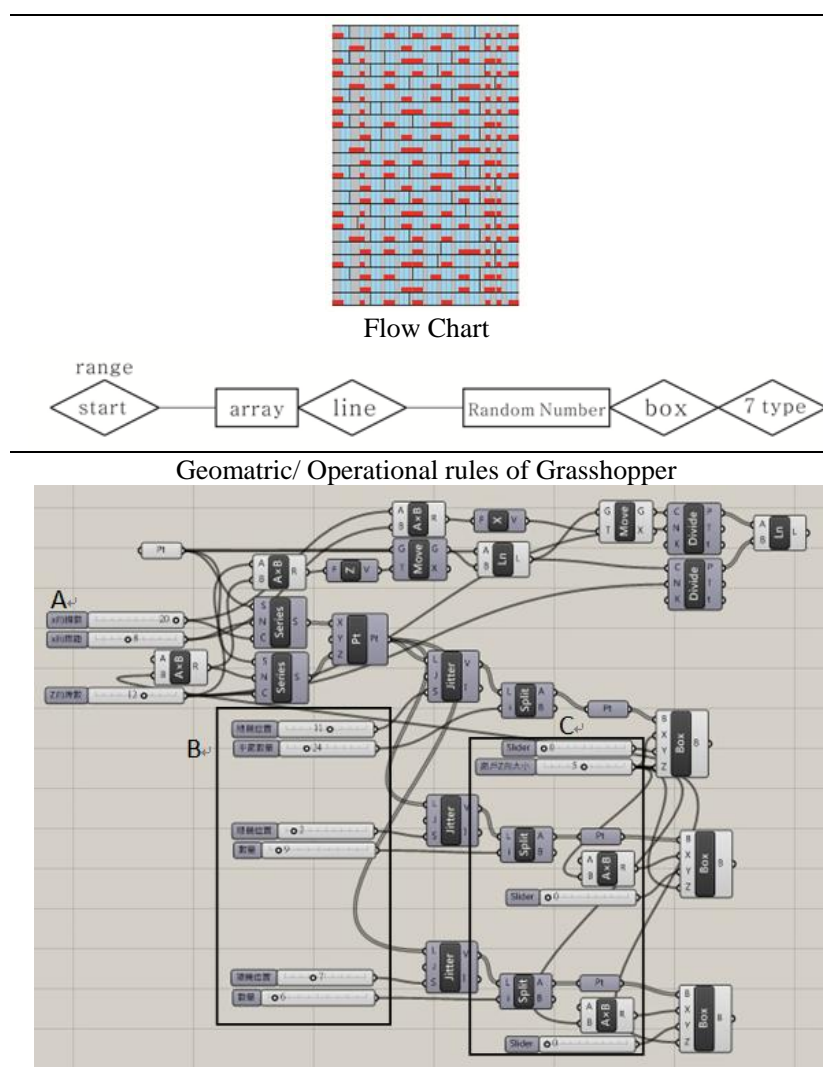

Test Patterns
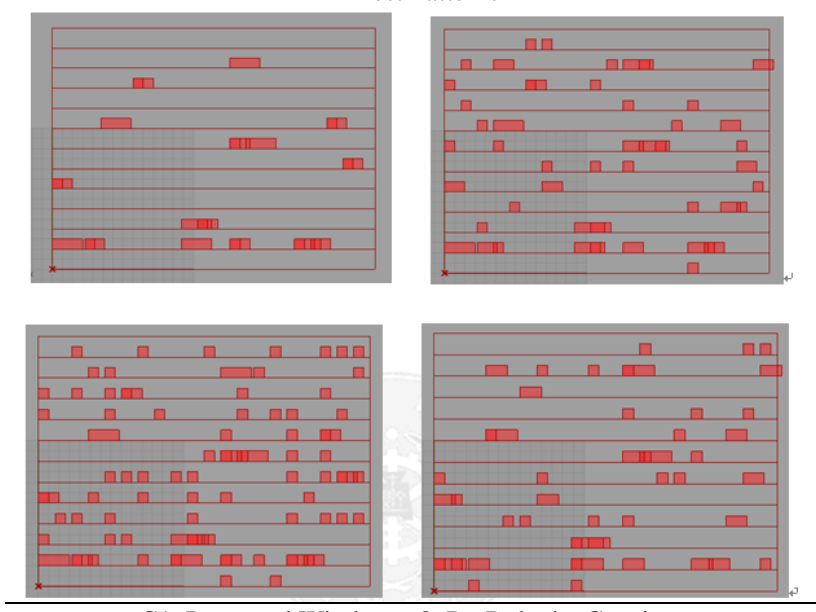

C1. Punctural Windows: 8. De Rokade, Groningen

Extraction of the pattern

20000

$\circ \bullet \circ$

00

100

100

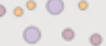

$\circ \circ 0$

000.

$\circ \circ 0$

两

-

- $\circ$

$\circ \circ$

$\circ \circ 0$

10

๑०

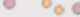

Flow Chart

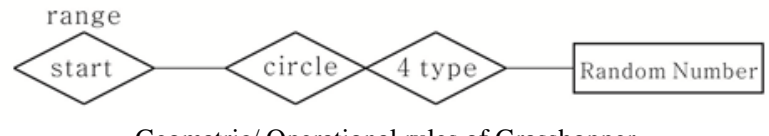

Geomatric/ Operational rules of Grasshopper 


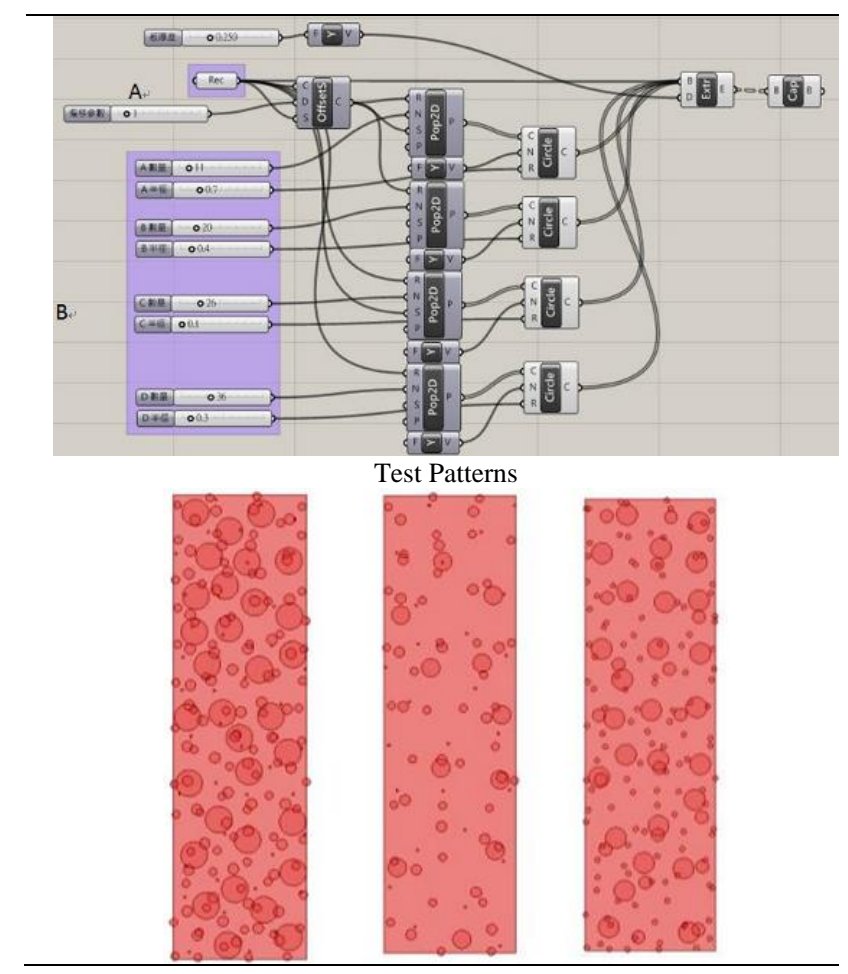

\section{DISCUSSION}

With the development of information technology, the process of building design shifts from drawing on paper to using computer systems where it is easier to integrate more data into facade design. With shape grammar and parametric software Grasshopper this research creates a spatial framework system, which can first model basic forms of facades of buildings and then can analyze the suitable facade elements and items according to the aesthetic and climatic conditions by rules.

However, the key purpose of these parametric models is not to copy the existing facade patterns but to provide a mechanism by which elements and items on the facades can be modelled, parameterized and created according to the conditions of its surrounding and architects. In this research we tried to model the patterns of openings and classify them into four kinds, such as: 1) balcony and terrace, 2) solar, louver and perforated panel, 3) window, 4) others. Then we set parameters for controlling the width, the length and the depth of an opening. We chose to focus on architectural openings because they are comparable to the eyes, noses, and ears in a human face, constituting the main characteristics of the facades of these high-rise residential buildings. For those building facades whose main concept shifts to the combination of materials or frames, there should be parameters to control the size and the combinations of the materials and the frames.

By controlling different measurements and inputs to adjust the parameters, the model system can then generate various facade proposals. Using these established models, researchers can analyze each single item to do more detailed study. Architects can also apply and modify each of these items to construct facades for other buildings through various combinations and permutations by changing its parameters. From a professional perspective the most suitable facade can be easily chosen from these facade proposals; and from a research perspective the relevant facade item model can be tested using more data from climate or material conditions.
The other issue is how to combine different facade items to a facade pattern. The impression of a facade is actually a holistic phenomenon. If we only focus on one single item of a facade, then the holistic concept of a facade will be ignored. Therefore architects and designers still have to create their own concepts for the facade designs and maybe check and modify the facade elements on their building facades by these parametric models. These digital models are only a tool for architects but not able to promise an excellent facade design.

\section{CONCLUSION}

This research chose eight features of facade skins from eight high-rise residential buildings, created a spatial framework system using the theory of shape grammar, and built up digital models using parametric factors on the facades by software Grasshopper. We also demonstrated here how to build up the rules that govern the basic forms of high-rise buildings, how to extract the patterns of the elements from these high-rise residential buildings and how to derive proposals from parametric models for facade design. Primarily the mechanism of modelling and deriving facade proposals is provided in this research. Thus, the goal of this research, to provide a mechanism for generating design proposals from a prototype and to facilitate the design process of various facade skins of high-rise residential buildings, has been reached. This also provides a mutual and vital framework to researchers and designers to go deeper into any issues with building facades.

\section{REFERENCES}

[1] C. Schittich, "Building skins," Institute for International Documentation, Munich, 2006.

[2] J. P. Burochin, O. Tournaire, and N. Paparoditis, "An unsupervised Hierarchical segmentation of a facade building image in elementary 2D- Models," presented at ISPRS Workshop on Object Extraction for 3D City Models, Road Databases and Traffic Monitoring, Paris, France, September 3- 4, 2009.

[3] P. Müller, G. Zeng, P. Wonka, and L. V. Gool, "Image-based Procedural Modeling of Facades," ACM Transactions on Graphic, vol. 26, no. 3, 2007.

[4] B. Hohmann, U. Krispel, S. Havemann, and D. Fellner, "City fit: High-quality urban reconstructions by fitting shape grammars to images and derived textured point clouds," in Proc. the Conference on International Congress of Arctic Social Science, July 14-19, 2008.

[5] A. F. Dellaert, "A probabilistic approach to the semantic interpretation of building facades," in Proc. the CIPA International Workshop on Vision Techniques Applied to the Rehabilitation of City Centre, October 25-27, Lisbon, Portugal, 2004.

[6] C. Brenner and N. Ripperda, "Extraction of Facades Using RJMCMC and Constraint Equations," in Proc. the ISPRS Commission lll Symposium on Photogrammetric and Computer Vision, Bonn, Germany, 2006.

[7] C. Brenner and N. Ripperda, "Application of a formal grammar to facade reconstruction in semiautomatic and automatic environments," in Proc. the 12th AGILE International Conference on Geographic Information Science 2009, Leibniz University Hannover, Germany, June 2-5, 2009.

[8] L. Quan, "Facade modeling," Image-Based Modeling, Springer Science+ Business Media, pp 177-198, 2010.

[9] C. H. Shen, S. S. Huang, H. Fu, and S. M. Hu, "Adaptive partitioning of urban facades," ACM Transaction on Graphic, vol.30, no. 6, article 184, December 2011.

[10] H. Riemenschneider, U. Krispel, W. Thaller, D. Michael, S. Havemann, D. Fellner, and B. Horst, "Irregular lattices for complex shape grammar facade parsing," in Proc. the 2012 IEEE Conference Computer Vision and Pattern Recognition, , June 16-21, 2012.

[11] J. Frazer, "Evolutionary architecture," Architectural Association, 1995. 


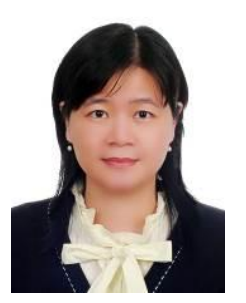

Yuchen S. Sung was born in Taipei, Taiwan. She completed her Ph.D. in the institute of Art History, Archaeology and Preservation, at Vienna University of Technology in 2006

She is an associate professor in the Architecture Department at Feng Chia University, Taichung, Taiwan. She has published several papers on shape grammar, such as "A study of spatial hierarchy of martial arts halls in taiwan," "Interpretation by

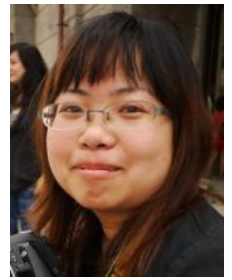

Yingjui Tseng was a research student in the Architecture Department at Feng Chia University, Taichung, 40724 Taiwan. She just graduated from the Architecture Department, Feng Chia University with a master's degree. grammars- the forms and functions of taiwanese railroad stations during Japanese colonial period," and "The spatial patterns and development of sugar industry cities- huwei and siny ing". She is also a practicing architect, although she does not have time for practicing right now. 